\title{
Blow up of solutions for 3D quasi-linear wave equations with positive initial energy
}

\section{AMIR PEYRAVI}

\begin{abstract}
.
In this paper we investigate blow up property of solutions for a system of nonlinear wave equations with nonlinear dissipations and positive initial energy in a bounded domain in $\mathbb{R}^{3}$. Our result improves and extends earlier results in the literature such as the ones in [Zhou, J. and $\mathrm{Mu}, \mathrm{C}$., The lifespan for 3D quasilinear wave equations with nonlinear damping terms, Nonlinear Anal., 74 (2011), 5455-5466] and [Pişkin, E., Uniform decay and blow-up of solutions for coupled nonlinear Klein-Gordon equations with nonlinear damping terms, Math. Meth. Appl. Scie., 37 (2014), No. 18, 3036-3047] in which the nonexistence results obtained only for negative initial energy or the one in [Ye, Y., Global existence and nonexistence of solutions for coupled nonlinear wave equations with damping and source terms, Bull. Korean Math. Soc., 51 (2014), No. 6, 1697-1710] where blow up results have been not addressed. Estimate for the lower bound of the blow up time is also given.
\end{abstract}

\section{REFERENCES}

[1] Agre, K. and Rammaha, M. A., Systems of nonlinear wave equations with damping and source terms, Differential Integral Equations., 19 (2006), No. 11, 1235-1270

[2] Alves, C. O., Cavalcanti, M. M., Cavalcanti, V. N. D., Rammaha, M. and Toundykov, D., On existence, uniform decay rates and blow up for solutions of systems of nonlinear wave equations with damping and source terms, Discr. Cont. Dyn. Syst. Ser. S, 2 (2009), 583-608

[3] Han, X. S. and Wang, M. X., Global existence and blow up of solutions for a system of nonlinear viscoelastic wave equations with damping and source, Nonlinear Anal., 71 (2009), 5427-5450

[4] Jörgens, K., Nonlinear wave equations, Department of Mathematics, University of Colorado, 1970

[5] Kafini, M. and Messaoudi, S. A., A blow-up result in a system of nonlinear viscoelastic wave equations with arbitrary positive initial energy, Indag. Math., 24 (2013), 602-612

[6] Li, G., Sun, Y. and Liu, W., Global existence, uniform decay and blow up of solutions for a system of Petrovsky equations, Nonlinear Anal., 74 (2011), 1523-1538

[7] Li, M. R. and Tsai, L. Y., Existence and nonexistence of global solutions of some systems of semilinear wave equations, Nonlinear Anal., 54 (2003), 1397-1415

[8] Li, M. R. and Tsai, L. Y., On a system of nonlinear wave equations, Taiwanese J. Math., 7 (2003), No. 4, 557-573

[9] Lili, S., Guo, B. and Gao, W., A lower bound for the blow-up time to a damped semilinear wave equation, Appl. Math. Lett., 37 (2014), 22-25

[10] Liu, W., Uniform decay of solutions for a quasilinear system of viscoelastic equations, Nonlinear Anal., 71 (2009), 2257-2267

[11] Liu, W. and Yu, J., Global Existence and Uniform Decay of Solutions for a Coupled System of Nonlinear Viscoelastic Wave Equations with Not Necessarily Differentiable Relaxation Functions, Stud. Appl. Math., 127 (2011), 315-344

[12] Liu, W. and Chen, K., Existence and general decay for nondissipative distributed systems with boundary frictional and memory dampings and acoustic boundary conditions, Z. Angew. Math. Phys., 66 (2015), 1595-1614

[13] Makhankov, V. G., Dynamics of classical solutions in integrable systems, Phys. Rep. (Sect. C Phys. Lett.), 35 (1978), 1-128

[14] Messaoudi, S. A. and Said-Houari, B., Global nonexistence of positive initial-energy solutions of a system of nonlinear viscoelastic wave equations with damping and source terms, J. Math. Anal. Appl., 365 (2010), 277-287

Received: 20.08.2015; In revised form: 15.04.2016; Accepted: 29.06.2016

2010 Mathematics Subject Classification. 35B44, 35L70, 35L52. time.

Key words and phrases. 3D quasilinear wave equations, nonlinear dissipation, blow up, lower bound for blow up 
[15] Medeiros, L. A. and Miranda, M. M., Weak solutions for a system of nonlinear Klein-Gordon equations, Ann. Mat. Pura Appl., CXLVI (1987), 173-183

[16] Medeiros, L. A. and Perla Menzala, G., On a mixed problem for a class of nonlinear Klein-Gordon equations, Acta Math. Hungar., 52 (1988), 61-69

[17] Miranda, M. M. and Medeiros, L. A., On the existence of global solutions of a coupled nonlinear Klein-Gorden equations, Funkcial. Ekvac., 30 (1987), 147-161

[18] Pişkin, E. Uniform decay and blow-up of solutions for coupled nonlinear Klein-Gordon equations with nonlinear damping terms, Math. Meth. Appl. Scie., 37 (2014), No. 18, 3036-3047

[19] Philipin, G. A., Lower bounds for blow-up time in a class of nonlinear wave equations, Z. Angew. Math. Phys., 66 (2015), No. 1, 129-134

[20] Reed, M., Abstract Nonlinear Wave Equations, Springer, Berlin, 1976

[21] Said-Houari, B., Global nonexistence of positive initial-energy solutions of a system of nonlinear wave equations with damping and source terms, Differential Integral Equations., 23 (2010), No. 1-2, 79-92

[22] Said-Houari, B., Exponential growth of positive initial-energy solutions of a system of nonlinear viscoelastic wave equations with damping and source terms, Z. Angew. Math. Phys., 62 (2011), No. 1, 115-133

[23] Said-Houari, B., Messaoudi, S. A. and Guesmia, A., General decay of solutions of a nonlinear system of viscoelastic wave equations, NoDEA Nonlinear Differential Equations Appl., 18 (2011), No. 6, 659-684

[24] Segal, I., Nonlinear partial differential equations in quantum field theory, 1965, Proc. Sympos. Appl. Math., Vol. XVII pp. 210-226 Amer. Math. Soc., Providence, R. I.

[25] Talenti, G., Best constant in Sobolev inequality, Ann. Mat. Pura Appl., 110 (1976), 353-372

[26] Wu, S. T., Blow-up results for systems of nonlinear Klein-Gordon equations with arbitrary positive initial energy, Electron. J. Differential Equations., 2012 (2012), No. 92, 1-13

[27] Wu, S. T., General decay of solutions for a nonlinear system of viscoelastic wave equations with degenerate damping and source terms, J. Math. Anal. Appl., 406 (2013), No. 1, 34-48

[28] Ye, Y., Global existence and asymptotic stability for coupled nonlinear Klein-Gordon equations with nonlinear damping terms, Dyn. Syst., 28 (2013), No. 2, 287-298

[29] Ye, Y., Global existence and nonexistence of solutions for coupled nonlinear wave equations with damping and source terms, Bull. Korean Math. Soc., 51 (2014), No. 6, 1697-1710

[30] Zhou, J., Lower bounds for blow-up time of two nonlinear wave equations, Appl. Math. Lett., 45 (2015), 64-68

[31] Zhou, J. and Mu, C., The lifespan for 3D quasilinear wave equations with nonlinear damping terms, Nonlinear Anal., 74 (2011), 5455-5466

\author{
DEPARTMENT OF MATHEMATICS \\ COLLEGE OF SCIENCES \\ SHIRAZ UNIVERSITY \\ SHIRAZ, 71467-13565, IRAN \\ E-mail address: peyravieshirazu.ac.ir
}

